\title{
On the relation between conformally invariant operators and some geometric tensors
}

\author{
Paolo Mastrolia and Dario D. Monticelli
}

\begin{abstract}
In this note we introduce and study some new tensors on general Riemannian manifolds which provide a link between the geometry of the underlying manifold and conformally invariant operators (up to order four). We study some of their properties and their relations with wellknown geometric objects, such as the scalar curvature, the $Q$-curvature, the Paneitz operator and the Schouten tensor, and with the elementary conformal tensors $\left\{T_{m, \alpha}^{u}\right\}$ and $\left\{X_{m, \mu}^{u}\right\}$ on Euclidean space introduced in [7] and [6].
\end{abstract}

\section{Introduction and statement of results}

In [7] and [6] we have recently given (in collaboration with Y. Y. Li) a complete characterization of fully nonlinear conformally invariant differential operators of any integer order on $\mathbb{R}^{n}$, which extends the result proved for second order operators by $\mathrm{A}$. Li and Y. Y. Li in [5]. In particular, there we proved existence and uniqueness of families of tensors, which we denote by $\left\{T_{m, \alpha}^{u}\right\}$ and $\left\{X_{m, \mu}^{u}\right\}$, for $m \in \mathbb{N}, m \geq 2$ and $\alpha, \mu \neq 0$, that are suitably invariant under Möbius transformations and that are the basic building blocks appearing in the definition of all conformally invariant differential operators on $\mathbb{R}^{n}$; we also explicitly computed the tensors that are related to operators of order up to four. We refer the interested reader to Definition 1.6 and Theorems 1.13 and 1.16 in [7] for the elementary conformal tensors $T_{m, \alpha}^{u}$ of degree $\alpha \neq 0$, and to Definition 1.7 and Theorems 1.11 and 1.15 in [6] for the elementary conformal tensors of exponential type $X_{m, \mu}^{u}$ with exponent $\mu \neq 0$.

In this note we want to highlight the geometric relevance of the aforementioned results: to this end, we consider a general Riemannian manifold $(M, g)$ of dimension $n \geq 3$ and we introduce the three main objects which provide the link between our previous analysis and the geometry of the underlying manifold.

Mathematics Subject Classification (2010): Primary 58J70; Secondary 35G20,53C21.

Keywords: Fully nonlinear higher order equations, conformally invariant operators, Schouten tensor, Paneitz operator, $Q$-curvature, elementary conformal tensors. 
In what follows we will use the moving frame notation with respect to a local orthonormal coframe (see e.g. [9]): the Einstein summation convention will be in force throughout, and we fix the index range $1 \leq i, j, \ldots \leq n$. Moreover we shall use the subscript $g$ to indicate the dependence of an object on the metric $g$.

Henceforth we shall denote by $\operatorname{Hess}_{g}(f)$ the Hessian of a $C^{2}$-function $f$ on $M$, by $\mathrm{Ric}_{g}$ the Ricci curvature tensor of the manifold, by $S_{g}$ its scalar curvature and by $\mathcal{A}_{g}=\frac{1}{n-2}\left(\operatorname{Ric}_{g}-\frac{S_{g}}{2(n-1)} g\right)$ the Schouten tensor.

We denote by $\left\{\theta^{i}\right\}, i=1, \ldots, n=\operatorname{dim} M$ a local orthonormal coframe on $(M, g)$, so that, for instance, we have $\operatorname{Ric}_{g}=R_{i j} \theta^{j} \otimes \theta^{i}$ and $\mathcal{A}_{g}=A_{i j} \theta^{j} \otimes \theta^{i}$. We also denote by $\mathcal{A}_{g}^{2}$ the symmetric $(0,2)$-tensor

$$
\mathcal{A}_{g}^{2}=\left(\mathcal{A}_{g}^{\sharp} \circ \mathcal{A}_{g}^{\sharp}\right)^{b},
$$

with (local) components $\left(A^{2}\right)_{i j}=\left(A_{i t} A_{t j}\right)$, and where $\sharp$ and $b$ are the usual musical isomorphisms. Finally, we recall that, for $n \geq 5$, the Paneitz operator on $(M, g)$ is defined by (see for instance [3] or [1])

$$
P_{g} u=\Delta_{g}^{2} u-\operatorname{div}_{g}\left\{a_{n} S_{g} d u+b_{n} \operatorname{Ric}_{g}\left(\nabla_{g} u, \cdot\right)\right\}+\frac{n-4}{2} Q_{g} u,
$$

where $\operatorname{div}_{g}$ is the divergence, $a_{n}=\frac{(n-2)^{2}+4}{2(n-1)(n-2)}, b_{n}=-\frac{4}{n-2}$ and $Q_{g}$ is the $Q$-curvature, which for $n \geq 4$ can be written (see e.g. [1]) as

$$
Q_{g}=-\frac{\Delta_{g} S_{g}}{2(n-1)}-\frac{2\left|\mathrm{Ric}_{g}\right|_{g}^{2}}{(n-2)^{2}}+\frac{n^{3}-4 n^{2}+16 n-16}{8(n-1)^{2}(n-2)^{2}} S_{g}^{2} .
$$

When $n=4$ the Paneitz operator and the $Q$-curvature have respectively the forms $P_{g} u=\Delta_{g}^{2} u-\operatorname{div}_{g}\left\{\frac{2}{3} S_{g} d u-2 \operatorname{Ric}_{g}\left(\nabla_{g} u, \cdot\right)\right\}, \quad Q_{g}=-\frac{1}{6} \Delta_{g} S_{g}-\frac{1}{2}\left|\operatorname{Ric}_{g}\right|_{g}^{2}+\frac{1}{6} S_{g}^{2}$.

The main character in our results is the following tensor of type $(0,4)$, which is defined on a generic Riemannian manifold $(M, g)$ with $n=\operatorname{dim} M \geq 3$.

Definition 1.1. We define the tensor $Z_{g}=Z_{i j k t} \theta^{t} \otimes \theta^{k} \otimes \theta^{j} \otimes \theta^{i}$ by

$$
\begin{aligned}
Z_{g}= & \nabla_{g}^{2} \mathcal{A}_{g}-\frac{n}{2}\left\{\mathcal{A}_{g} \otimes \mathcal{A}_{g}+\left[A_{j t} A_{i k}+A_{i t} A_{j k}\right] \theta^{t} \otimes \theta^{k} \otimes \theta^{j} \otimes \theta^{i}\right\} \\
& +\left\{\mathcal{A}_{g}^{2} \otimes g+\left[\left(A^{2}\right)_{j t} \delta_{i k}+\left(A^{2}\right)_{i t} \delta_{j k}\right] \theta^{t} \otimes \theta^{k} \otimes \theta^{j} \otimes \theta^{i}\right\}
\end{aligned}
$$

where $\nabla_{g}^{2} \mathcal{A}_{g}$ is the second covariant derivative of the Schouten tensor with respect to the Levi-Civita connection associated with $g$. In components we have

$$
Z_{i j k t}=A_{i j, k t}-\frac{n}{2}\left[A_{k t} A_{j i}+A_{j t} A_{k i}+A_{i t} A_{j k}\right]+\left(A^{2}\right)_{k t} \delta_{j i}+\left(A^{2}\right)_{t j} \delta_{k i}+\left(A^{2}\right)_{t i} \delta_{j k} .
$$

The other two tensors of geometric relevance are, respectively, $\nabla_{g} \mathcal{A}_{g}$, the covariant derivative of the Schouten tensor, and the Schouten tensor itself. 
The following results reveal the relevance of the tensor $Z_{g}$ for the geometry of the underlying manifold.

Theorem 1.2. The tensor $Z_{g}=Z_{i j k t} \theta^{t} \otimes \theta^{k} \otimes \theta^{j} \otimes \theta^{i}$ defined in (1.3) satisfies

$$
Z_{i j k t}=Z_{j i k t}, \quad Z_{i j k t}=Z_{i k j t}+\frac{1}{n-2} C_{i j k, t},
$$

where $C_{g}=C_{i j k} \theta^{k} \otimes \theta^{j} \otimes \theta^{i}$ is the Cotton tensor and $C_{i j k, t}$ are the (local) components of its covariant derivative. Moreover, all the complete contractions of the tensor $Z_{g}$ give the opposite of the $Q$-curvature, i.e.,

$$
Z_{i i t t}=Z_{i t i t}=Z_{t i i t}=-Q_{g}
$$

The following proposition shows how $Z_{g}$ changes under the right (in a suitable sense, see Remark 1.6) conformal change of the metric.

Proposition 1.3. If $n \neq 4$ and $\tilde{g}=u^{4 /(n-4)} g$, then the (local) components of the tensor $Z_{\tilde{g}}=\tilde{Z}_{i j k t} \tilde{\theta}^{t} \otimes \tilde{\theta}^{k} \otimes \tilde{\theta}^{j} \otimes \tilde{\theta}^{i}$ satisfy

$$
\begin{aligned}
& u^{8 /(n-4)} \tilde{Z}_{i j k t}=Z_{i j k t}-\frac{2}{n-4} \frac{1}{u} u_{i j k t} \\
& +\frac{1}{u}\left[\frac{2}{n-4}\left(u_{l} A_{l i, t} \delta_{j k}+u_{l} A_{l j, t} \delta_{i k}+u_{l} A_{l i, k} \delta_{j t}+u_{l} A_{l j, k} \delta_{i t}+u_{l} A_{i j, l} \delta_{k t}\right)\right. \\
& -\frac{2}{n-4}\left(u_{i} A_{j k, t}+u_{j} A_{i k, t}+u_{i} A_{j t, k}+u_{j} A_{i t, k}+3 u_{k} A_{i j, t}+3 u_{t} A_{i j, k}\right) \\
& +A_{i j} u_{k t}+\frac{n-2}{n-4}\left(u_{i t} A_{j k}+u_{j t} A_{i k}\right)+\frac{n}{n-4}\left(u_{j k} A_{i t}+u_{i k} A_{j t}+u_{i j} A_{k t}\right) \\
& \left.-\frac{2}{n-4}\left(u_{l t} A_{k l} \delta_{i j}+u_{i l} A_{l t} \delta_{j k}+u_{j l} A_{l t} \delta_{i k}+u_{k l} A_{l t} \delta_{i j}\right)\right] \\
& +\frac{1}{u^{2}}\left[\frac{2(n+2)}{(n-4)^{2}}\left(u_{i j k} u_{t}+u_{i j t} u_{k}\right)+\frac{2 n}{(n-4)^{2}}\left(u_{j k t} u_{i}+u_{i k t} u_{j}\right)\right. \\
& +\frac{4}{(n-4)^{2}}\left(u_{t j k} u_{i}+u_{t i k} u_{j}\right)-\frac{8}{(n-4)^{2}}\left(u_{l} A_{l i} u_{t} \delta_{j k}+u_{l} A_{l j} u_{t} \delta_{i k}\right) \\
& -\frac{n^{2}-4 n-8}{(n-4)^{2}}\left(u_{i} u_{t} A_{j k}+u_{j} u_{t} A_{i k}\right)-\frac{n^{2}-6 n-16}{(n-4)^{2}} u_{k} u_{t} A_{i j} \\
& -\frac{n(n-2)}{(n-4)^{2}}\left(A_{i t} u_{j} u_{k}+A_{j t} u_{i} u_{k}+A_{k t} u_{i} u_{j}\right)+\frac{2}{n-4}\left(u_{l} A_{l t} u_{i} \delta_{j k}+u_{l} A_{l t} u_{j} \delta_{i k}\right) \\
& +\frac{2(n-2)}{(n-4)^{2}}\left(u_{l} u_{t} A_{k l} \delta_{i j}+u_{l} u_{k} A_{l t} \delta_{i j}\right)+\frac{4}{(n-4)^{2}} \mathcal{A}_{g}\left(\nabla_{g} u, \nabla_{g} u\right)\left(\delta_{i t} \delta_{j k}+\delta_{i k} \delta_{j t}\right) \\
& -\frac{4}{(n-4)^{2}}\left(u_{l} u_{l k t} \delta_{i j}+u_{l} u_{l j t} \delta_{i k}+u_{l} u_{l i t} \delta_{j k}+u_{l} u_{l j k} \delta_{i t}+u_{l} u_{l i k} \delta_{j t}+u_{l} u_{i j l} \delta_{k t}\right)
\end{aligned}
$$




$$
\begin{aligned}
& +\frac{4}{(n-4)^{2}}\left(2 u_{i} u_{j} A_{k t}+3 u_{i} u_{k} A_{j t}+3 u_{j} u_{k} A_{i t}\right)+\frac{n-8}{(n-4)^{2}}\left|\nabla_{g} u\right|_{g}^{2} A_{i j} \delta_{k t} \\
& +\frac{\left|\nabla_{g} u\right|_{g}^{2}}{n-4}\left(A_{j k} \delta_{i t}+A_{i k} \delta_{j t}+A_{i t} \delta_{j k}+A_{j t} \delta_{i k}+A_{k t} \delta_{i j}\right)-\frac{4}{(n-4)^{2}}\left(u_{i} u_{l} A_{l k} \delta_{j t}\right. \\
& \left.\left.+u_{j} u_{l} A_{l k} \delta_{i t}+u_{i} u_{l} A_{l j} \delta_{k t}+u_{j} u_{l} A_{l i} \delta_{k t}+2 u_{k} u_{l} A_{l j} \delta_{i t}+2 u_{k} u_{l} A_{l i} \delta_{j t}\right)\right] \\
& +\frac{1}{u^{3}}\left[\frac { 4 n } { ( n - 4 ) ^ { 3 } } \left(u_{l} u_{l k} u_{t} \delta_{i j}+u_{l} u_{l j} u_{t} \delta_{i k}+u_{l} u_{l i} u_{t} \delta_{j k}+u_{l} u_{l t} u_{i} \delta_{j k}+u_{l} u_{l t} u_{j} \delta_{i k}\right.\right. \\
& \left.+u_{l} u_{l t} u_{k} \delta_{i j}\right)-\frac{2 n(n+2)}{(n-4)^{3}}\left(u_{i} u_{t} u_{j k}+u_{j} u_{t} u_{i k}+u_{k} u_{t} u_{i j}+u_{i} u_{j} u_{k t}+u_{i} u_{k} u_{j t}\right. \\
& \left.+u_{j} u_{k} u_{i t}\right)+\frac{2 n\left|\nabla_{g} u\right|_{g}^{2}}{(n-4)^{3}}\left(u_{i t} \delta_{j k}+u_{j t} \delta_{i k}+u_{k t} \delta_{i j}+u_{i j} \delta_{k t}+u_{i k} \delta_{j t}+u_{j k} \delta_{i t}\right) \\
& -\frac{8}{(n-4)^{3}} \operatorname{Hess}_{g}(u)\left(\nabla_{g} u, \nabla_{g} u\right)\left(\delta_{i j} \delta_{k t}+\delta_{i k} \delta_{j t}+\delta_{j k} \delta_{i t}\right)+\frac{4 n}{(n-4)^{3}}\left(u_{i} u_{l} u_{l k} \delta_{j t}\right. \\
& \left.\left.+u_{j} u_{l} u_{l k} \delta_{i t}+u_{i} u_{l} u_{l j} \delta_{k t}+u_{j} u_{l} u_{l i} \delta_{k t}+u_{k} u_{l} u_{l i} \delta_{j t}+u_{k} u_{l} u_{l j} \delta_{i t}\right)\right] \\
& +\frac{1}{u^{4}}\left[\frac{6 n(n+2)(n-2)}{(n-4)^{4}} u_{i} u_{j} u_{k} u_{t}\right. \\
& -\frac{6 n(n-2)}{(n-4)^{4}}\left|\nabla_{g} u\right|_{g}^{2}\left(u_{i} u_{t} \delta_{j k}+u_{j} u_{t} \delta_{i k}+u_{k} u_{t} \delta_{i j}+u_{i} u_{j} \delta_{k t}+u_{i} u_{k} \delta_{j t}+u_{j} u_{k} \delta_{i t}\right) \\
& \left.+\frac{6(n-2)}{(n-4)^{4}}\left|\nabla_{g} u\right|_{g}^{4}\left(\delta_{i j} \delta_{k t}+\delta_{i k} \delta_{j t}+\delta_{j k} \delta_{i t}\right)\right] \text {. }
\end{aligned}
$$

Now we can tie $Z_{g}$ both to the $Q$-curvature and the Paneitz operator and to the elementary conformal tensors $\left\{T_{m, \alpha}^{u}\right\}$ and $\left\{X_{m, \mu}^{u}\right\}$ on Euclidean space through the following corollaries.

Corollary 1.4. If $(M, g)=\left(\mathbb{R}^{n}, g_{\text {flat }}\right)$, where $g_{\text {flat }}$ is the standard Euclidean metric, and $\tilde{g}=u^{4 /(n-4)} g_{\text {flat }}, n \neq 4$, then equation (1.7) becomes

$$
Z_{\tilde{g}}=-\frac{2}{n-4} u^{8 /(n-4)} T_{4,(n-4) /(2 n)}^{u} .
$$

Corollary 1.5. All the complete contractions of equation (1.7) yield

$$
u^{8 /(n-4)} Q_{\tilde{g}}=\frac{2}{n-4} \frac{1}{u} P_{g} u,
$$

which is equivalent to the $Q$-curvature equation on $(M, g)$, i.e.,

$$
P_{g} u=\frac{n-4}{2} Q_{\tilde{g}} u^{(n+4) /(n-4)} .
$$


In particular, if $(M, g)=\left(\mathbb{R}^{n}, g_{\text {flat }}\right)$, then $(1.9)$ becomes

$$
\Delta^{2} u=\frac{n-4}{2} Q_{\tilde{g}} u^{(n+4) /(n-4)} .
$$

Remark 1.6. Note that $\tilde{g}=u^{4 /(n-4)} g$ is the most natural conformal change of the original metric $g$ that is customarily used to exploit the invariance properties of the $Q$-curvature and of the Paneitz operator. One could also consider different conformal changes of the metric; see Proposition 1.10.

The case $n=4$ requires a conformal change of the underlying metric of exponential type, which produces the following results.

Proposition 1.7. If $n=4$ and $\tilde{g}=e^{2 u} g$, then the (local) components of the tensor $Z_{\tilde{g}}=\tilde{Z}_{i j k t} \tilde{\theta}^{t} \otimes \tilde{\theta}^{k} \otimes \tilde{\theta}^{j} \otimes \tilde{\theta}^{i}$ satisfy

$$
\begin{aligned}
& e^{4 u} \tilde{Z}_{i j k t}=Z_{i j k t}-u_{i j k t} \\
& +u_{l} A_{l i, t} \delta_{j k}+u_{l} A_{l j, t} \delta_{i k}+u_{l} A_{l i, k} \delta_{j t}+u_{l} A_{l j, k} \delta_{i t}+u_{l} A_{i j, l} \delta_{k t} \\
& -\left[u_{i} A_{j k, t}+u_{j} A_{i k, t}+u_{i} A_{j t, k}+u_{j} A_{i t, k}+3 u_{k} A_{i j, t}+3 u_{t} A_{i j, k}\right] \\
& +u_{j t} A_{i k}+u_{i t} A_{j k}+2 u_{j k} A_{i t}+2 u_{i k} A_{j t}+2 u_{i j} A_{k t} \\
& +2 u_{i} u_{j k t}+u_{i} u_{j t k}+2 u_{j} u_{i k t}+u_{j} u_{i t k}+3 u_{k} u_{i j t}+3 u_{t} u_{i j k} \\
& -\left[u_{l} u_{l k t} \delta_{i j}+u_{l} u_{l j t} \delta_{i k}+u_{l} u_{l i t} \delta_{j k}\right]+u_{i} u_{k} A_{j t}+u_{j} u_{k} A_{i t} \\
& -\left[u_{l t} A_{k l} \delta_{i j}+u_{i l} A_{l t} \delta_{j k}+u_{j l} A_{l t} \delta_{i k}+u_{k l} A_{l t} \delta_{i j}\right] \\
& -\left[2 u_{l} A_{l i} u_{t} \delta_{j k}+2 u_{l} A_{l j} u_{t} \delta_{i k}\right]+2\left[u_{i} u_{t} A_{j k}+u_{j} u_{t} A_{i k}+3 u_{k} u_{t} A_{i j}\right] \\
& +u_{l} u_{t} A_{k l} \delta_{i j}+u_{l} u_{k} A_{l t} \delta_{i j}-\left[u_{l} u_{l j k} \delta_{i t}+u_{l} u_{l i k} \delta_{j t}+u_{l} u_{i j l} \delta_{k t}\right] \\
& +\mathcal{A}_{g}\left(\nabla_{g} u, \nabla_{g} u\right)\left(\delta_{i t} \delta_{j k}+\delta_{i k} \delta_{j t}\right)-\left|\nabla_{g} u\right|_{g}^{2} A_{i j} \delta_{k t}-\left[2 u_{k} u_{l} A_{l j} \delta_{i t}+2 u_{k} u_{l} A_{l i} \delta_{j t}\right] \\
& -\left[u_{i} u_{l} A_{l k} \delta_{j t}+u_{j} u_{l} A_{l k} \delta_{i t}+u_{i} u_{l} A_{l j} \delta_{k t}+u_{j} u_{l} A_{l i} \delta_{k t}\right] \\
& +2\left[u_{l} u_{l k} u_{t} \delta_{i j}+u_{l} u_{l j} u_{t} \delta_{i k}+u_{l} u_{l i} u_{t} \delta_{j k}+u_{l} u_{l t} u_{i} \delta_{j k}+u_{l} u_{l t} u_{j} \delta_{i k}+u_{l} u_{l t} u_{k} \delta_{i j}\right. \\
& \left.+u_{i} u_{l} u_{l k} \delta_{j t}+u_{j} u_{l} u_{l k} \delta_{i t}+u_{i} u_{l} u_{l j} \delta_{k t}+u_{j} u_{l} u_{l i} \delta_{k t}+u_{k} u_{l} u_{l i} \delta_{j t}+u_{k} u_{l} u_{l j} \delta_{i t}\right] \\
& -6\left[u_{i} u_{t} u_{j k}+u_{j} u_{t} u_{i k}+u_{k} u_{t} u_{i j}+u_{i} u_{j} u_{k t}+u_{i} u_{k} u_{j t}+u_{j} u_{k} u_{i t}\right] \\
& +\left|\nabla_{g} u\right|_{g}^{2}\left[u_{i t} \delta_{j k}+u_{j t} \delta_{i k}+u_{k t} \delta_{i j}+u_{i j} \delta_{k t}+u_{i k} \delta_{j t}+u_{j k} \delta_{i t}\right] \\
& -\operatorname{Hess}_{g}(u)\left(\nabla_{g} u, \nabla_{g} u\right)\left(\delta_{i j} \delta_{k t}+\delta_{i k} \delta_{j t}+\delta_{j k} \delta_{i t}\right)+18 u_{i} u_{j} u_{k} u_{t} \\
& -3\left|\nabla_{g} u\right|_{g}^{2}\left(u_{i} u_{t} \delta_{j k}+u_{j} u_{t} \delta_{i k}+u_{k} u_{t} \delta_{i j}+u_{i} u_{j} \delta_{k t}+u_{i} u_{k} \delta_{j t}+u_{j} u_{k} \delta_{i t}\right) \\
& +\frac{3}{4}\left|\nabla_{g} u\right|_{g}^{4}\left(\delta_{i j} \delta_{k t}+\delta_{i k} \delta_{j t}+\delta_{j k} \delta_{i t}\right) \text {. }
\end{aligned}
$$

Corollary 1.8. If $(M, g)=\left(\mathbb{R}^{4}, g_{\text {flat }}\right)$ and $\tilde{g}=e^{2 u} g_{\text {flat }}$, then equation (1.10) becomes

$$
Z_{\tilde{g}}=-e^{4 u} X_{4,1 / 4}^{u}
$$


Corollary 1.9. All the complete contractions of equation (1.10) yield the $Q$-curvature equation on $\left(M^{4}, g\right)$, i.e.,

$$
P_{g} u+Q_{g}=e^{4 u} Q_{\tilde{g}}
$$

In particular, if $(M, g)=\left(\mathbb{R}^{4}, g_{\text {flat }}\right)$, then $(1.12)$ becomes

$$
\Delta^{2} u=e^{4 u} Q_{\tilde{g}}
$$

The next proposition shows how the tensor $Z_{g}$ on $\mathbb{R}^{n}$ changes under a generic conformal transformation (of polynomial or exponential type) of the underlying metric. This further connects the tensor $Z_{g}$ with the elementary conformal tensors $T_{m, \alpha}^{u}$ and $X_{m, \mu}^{u}$.

Proposition 1.10. If $(M, g)=\left(\mathbb{R}^{n}, g_{\text {flat }}\right)$ then $Z_{g}$ changes under a conformal transformation of polynomial type of the underlying metric, $\tilde{g}=u^{2 \beta} g_{\text {flat }}$, according to the formula

$$
\begin{aligned}
Z_{\tilde{g}}= & -\beta u^{4 \beta} T_{4,1 /(n \beta)}^{u}+\left(\frac{1}{\beta}-\frac{n-4}{2}\right) \beta^{2} u^{4 \beta}\left\{T_{2,1 /(n \beta)}^{u} \otimes T_{2,1 /(n \beta)}^{u}\right. \\
& \left.+\left[\left(T_{2,1 /(n \beta)}^{u}\right)_{t j}\left(T_{2,1 /(n \beta)}^{u}\right)_{k i}+\left(T_{2,1 /(n \beta)}^{u}\right)_{t i}\left(T_{2,1 /(n \beta)}^{u}\right)_{k j}\right] \theta^{t} \otimes \theta^{k} \otimes \theta^{j} \otimes \theta^{i}\right\} .
\end{aligned}
$$

On the other hand, $Z_{g}$ changes under a conformal transformation of exponential type of the underlying metric, $\tilde{g}=e^{2 \alpha u} g_{\text {flat }}$, according to the formula

$$
\begin{aligned}
Z_{\tilde{g}} & =-\alpha e^{4 \alpha u} X_{4,1 /(n \alpha)}^{u}+\frac{4-n}{2} \alpha^{2} e^{4 \alpha u}\left\{X_{2,1 /(n \alpha)}^{u} \otimes X_{2,1 /(n \alpha)}^{u}\right. \\
& \left.+\left[\left(X_{2,1 /(n \alpha)}^{u}\right)_{t j}\left(X_{2,1 /(n \alpha)}^{u}\right)_{k i}+\left(X_{2,1 /(n \alpha)}^{u}\right)_{t i}\left(X_{2,1 /(n \alpha)}^{u}\right)_{k j}\right] \theta^{t} \otimes \theta^{k} \otimes \theta^{j} \otimes \theta^{i}\right\} .
\end{aligned}
$$

The traces of the tensor $Z_{g}$ can now be used to formulate a fully nonlinear generalization of the $Q$-curvature problem on $(M, g)$, i.e., the problem of finding a metric $\tilde{g}$, conformally related to $g$, such that $Q_{\tilde{g}}$ is constant.

First we define the tensor $\mathcal{U}_{g}=U_{i j} \theta^{j} \otimes \theta^{i}$ with components

$$
\begin{aligned}
U_{i j} & =Z_{i j t t}=A_{i j, t t}-\frac{n}{2}\left(2 A_{i t} A_{t j}+A_{t t} A_{i j}\right)+2\left(A^{2}\right)_{i j}+\left(A^{2}\right)_{t t} \delta_{i j} \\
& =A_{i j, t t}-(n-2)\left(A^{2}\right)_{i j}-\frac{n}{2}\left(\operatorname{tr}_{g} \mathcal{A}_{g}\right) A_{i j}+\left|\mathcal{A}_{g}\right|_{g}^{2} \delta_{i j} .
\end{aligned}
$$

Note that the tensor $\mathcal{U}_{g}$ is symmetric, i.e., $U_{i j}=U_{j i}$ for every $i, j=1, \ldots, n$.

Next, we introduce the tensor $\mathcal{H}_{g}=H_{i j} \theta^{j} \otimes \theta^{i}$ with components

$$
\begin{aligned}
H_{i j} & =Z_{t i t j}=A_{t i, t j}-\frac{n}{2}\left(2 A_{i t} A_{t j}+A_{t t} A_{i j}\right)+2\left(A^{2}\right)_{i j}+n\left(A^{2}\right)_{i j} \\
& =A_{t t, i j}-\frac{n}{2}\left(\operatorname{tr}_{g} \mathcal{A}_{g}\right) A_{i j}+2\left(A^{2}\right)_{i j} .
\end{aligned}
$$

Note that for every $i, j=1, \ldots, n$ one has

$$
Z_{i t t j}=Z_{t i t j}=H_{i j}
$$


and

$$
Z_{t t i j}=A_{t t, i j}-\frac{n}{2}\left(2 A_{i t} A_{t j}+A_{t t} A_{i j}\right)+2\left(A^{2}\right)_{i j}+n\left(A^{2}\right)_{i j}=H_{i j} .
$$

Note also that we can write

$$
\mathcal{H}_{g}=\frac{1}{2(n-1)} \operatorname{Hess}_{g}\left(S_{g}\right)-\frac{n S_{g}}{4(n-1)} \mathcal{A}_{g}+2 \mathcal{A}_{g}^{2}
$$

and that $\mathcal{H}_{g}$ is symmetric, i.e., $H_{i j}=H_{j i}$ for every $i, j=1, \ldots, n$. Obviously one has

$$
\operatorname{tr}_{g} \mathcal{H}_{g}=\operatorname{tr}_{g} \mathcal{U}_{g}=-Q_{g}
$$

Since the tensors $\mathcal{U}_{g}$ and $\mathcal{H}_{g}$ are both symmetric, in the spirit of the wellknown $k$-Yamabe problem (see [10] and the references therein) we are led to the formulation of the following problems: find a metric in the conformal class of the original metric $g$ such that $\sigma_{k}\left(\mathcal{H}_{g}\right), k=1, \ldots, n$, is constant, or find a metric in the conformal class of the original metric $g$ such that $\sigma_{k}\left(\mathcal{U}_{g}\right), k=1, \ldots, n$, is constant, where $\sigma_{k}(\cdot)$ denotes the $k^{\text {th }}$ elementary symmetric function of the eigenvalues of the tensor in the argument.

These problems, by (1.13), can be considered as fourth order fully nonlinear generalizations of the $Q$-curvature problem.

Remark 1.11. Note that we could also define the tensor $\mathcal{Y}_{g}=Y_{i j} \theta^{j} \otimes \theta^{i}$ with components

$$
\begin{aligned}
Y_{i j} & =Z_{i t j t}=A_{i t, j t}-\frac{n}{2}\left(2 A_{i t} A_{t j}+A_{t t} A_{i j}\right)+2\left(A^{2}\right)_{i j}+\left(A^{2}\right)_{t t} \delta_{i j} \\
& =A_{i t, j t}-n\left(A^{2}\right)_{i j}-\frac{n}{2} A_{t t} A_{i j}+2\left(A^{2}\right)_{i j}+\left(A^{2}\right)_{t t} \delta_{i j} \\
& =A_{i j, t t}-\frac{1}{n-2} C_{i j t, t}-(n-2)\left(A^{2}\right)_{i j}-\frac{n}{2}\left(\operatorname{tr}_{g} \mathcal{A}_{g}\right) A_{i j}+\left|\mathcal{A}_{g}\right|_{g}^{2} \delta_{i j},
\end{aligned}
$$

and that one also has $Y_{i j}=Z_{t i j t}=Z_{i t j t}$. Although $\mathcal{Y}_{g}$ in general is not symmetric, i.e., $Y_{i j} \neq Y_{j i}$, we have that $\mathcal{U}_{g}=\mathcal{Y}_{g}$ if $\operatorname{div}_{g} C_{g}=0$ and that

$$
\operatorname{tr}_{g} \mathcal{Y}_{g}=\operatorname{tr}_{g} \mathcal{H}_{g}=\operatorname{tr}_{g} \mathcal{U}_{g}=-Q_{g}
$$

For tensors of order 2 and 3 , the relations between $\mathcal{A}_{g}$ and $\nabla_{g} \mathcal{A}_{g}$ and the elementary conformal tensors $T_{2, \alpha}^{u}, T_{3, \alpha}^{u}$ and $X_{2, \mu}^{u}, X_{3, \mu}^{u}$ take a simpler form.

Proposition 1.12. Let $(M, g)=\left(\mathbb{R}^{n}, g_{\text {flat }}\right)$. If $\tilde{g}=u^{2 \beta} g_{\text {flat }}, u \in C^{2}\left(\mathbb{R}^{n}\right), u>0$, $\beta \neq 0$ there holds:

$$
\mathcal{A}_{\tilde{g}}=-\beta u^{2 \beta} T_{2,1 /(n \beta)}^{u} .
$$

On the other hand, if $\tilde{g}=e^{2 \alpha u} g_{\text {flat }}, u \in C^{2}\left(\mathbb{R}^{n}\right), \alpha \neq 0$ then

$$
\mathcal{A}_{\tilde{g}}=-\alpha e^{2 \alpha u} X_{2,1 /(n \alpha)}^{u} .
$$


Proposition 1.13. Let $(M, g)=\left(\mathbb{R}^{n}, g_{\text {flat }}\right)$, where $g_{\text {flat }}$ denotes the Euclidean metric. If $\tilde{g}=u^{2 \beta} g_{\text {flat }}, u \in C^{3}\left(\mathbb{R}^{n}\right), u>0, \beta \neq 0$ then there holds:

$$
\nabla_{\tilde{g}} \mathcal{A}_{\tilde{g}}=-\beta u^{3 \beta} T_{3,1 /(n \beta)}^{u} .
$$

On the other hand, if $\tilde{g}=e^{2 \alpha u} g_{\text {flat }}, u \in C^{3}\left(\mathbb{R}^{n}\right), \alpha \neq 0$ then there holds:

$$
\nabla_{\tilde{g}} \mathcal{A}_{\tilde{g}}=-\alpha e^{3 \alpha u} X_{3,1 /(n \alpha)}^{u} .
$$

We conclude the section with two open questions. It would be interesting to construct higher order tensors related to higher order $Q$-curvatures and to the GJMS operators (see [4]), which are connected to them in the same way as the $(0,4)$-tensor $Z_{g}$ introduced in $(1.3)$ is connected to the $Q$-curvature and to the Paneitz operator. Of course, if possible at all, this construction must break down (on manifolds $\left(M^{n}, g\right)$ of even dimension) for tensors of even order greater than the dimension of $M$; again see [4]. We also note that in [8] a complete characterization of second order fully nonlinear CR invariant differential equations on the Heisenberg group was given, which is the analogue in the CR setting of the result proven by $\mathrm{A}$. Li and Y. Y. Li in [5] in the Euclidean case. One could try to construct also the CR analogue on the Heisenberg group of the elementary conformal tensors $\left\{T_{m, \alpha}^{u}\right\}$ and $\left\{X_{m, \mu}^{u}\right\}$ defined on $\mathbb{R}^{n}$, and the analogue in the CR setting of the tensor $Z_{g}$ defined in (1.3) on a general Riemannian manifold.

\section{Sketch of the proofs of the results}

Using the moving frame formalism most of the proofs consist in quite straightforward but long and tedious calculations; in order to keep the length of the paper reasonable we will just give an idea of to prove most of them.

Proof of Theorem 1.2. Using equation (1.4), the symmetry of $Z_{g}$ in the first two indices follows immediately from the symmetry of the Schouten tensor $\mathcal{A}_{g}$, i.e., $A_{i j}=A_{j i}$, and from the symmetry of $\mathcal{A}_{g}^{2}$.

Formula (1.5) follows using the definition of the Cotton tensor and observing that

$$
Z_{i j k t}-Z_{i k j t}=A_{i j, k t}-A_{i k, j t}
$$

From equation (1.4), noting that

$$
\begin{aligned}
A_{i t} A_{i t} & =\left|\mathcal{A}_{g}\right|_{g}^{2}=\frac{1}{(n-2)^{2}}\left[\left|\operatorname{Ric}_{g}\right|_{g}^{2}-\frac{3 n-4}{4(n-1)^{2}} S_{g}^{2}\right], \\
A_{i i} & =\operatorname{tr}_{g}\left(\mathcal{A}_{g}\right)=\frac{S_{g}}{2(n-1)}, \quad \text { and } \\
A_{i i, t t} & =\frac{\Delta_{g} S_{g}}{2(n-1)},
\end{aligned}
$$


we deduce

$$
\begin{aligned}
Z_{i i t t}= & \frac{\Delta_{g} S_{g}}{2(n-1)}-\frac{n}{2}\left[\frac{2\left|\operatorname{Ric}_{g}\right|_{g}^{2}}{(n-2)^{2}}+\frac{n^{2}-10 n+12}{4(n-1)^{2}(n-2)^{2}} S_{g}^{2}\right] \\
& +\frac{n+2}{(n-2)^{2}}\left[\left|\operatorname{Ric}_{g}\right|_{g}^{2}-\frac{3 n-4}{4(n-1)^{2}} S_{g}^{2}\right] \\
= & \frac{\Delta_{g} S_{g}}{2(n-1)}+\frac{2\left|\operatorname{Ric}_{g}\right|_{g}^{2}}{(n-2)^{2}}-\frac{n^{3}-4 n^{2}+16 n-16}{8(n-1)^{2}(n-2)^{2}} S_{g}^{2}=-Q_{g}
\end{aligned}
$$

The equality among all the complete contractions of $Z_{g}$ is a simple computation.

Proof of Propositions 1.3 and 1.7. We observe that, with respect to the (local) basis $\left\{\tilde{\theta}^{t} \otimes \tilde{\theta}^{k} \otimes \tilde{\theta}^{j} \otimes \tilde{\theta}^{i}\right\}, i, j, k, t=1, \ldots, n$, the components of $Z_{\tilde{g}}$ can be written as

$\tilde{Z}_{i j k t}=\tilde{A}_{i j, k t}-\frac{n}{2}\left(\tilde{A}_{k t} \tilde{A}_{j i}+\tilde{A}_{t j} \tilde{A}_{i k}+\tilde{A}_{i t} \tilde{A}_{j k}\right)+\left(\tilde{A}^{2}\right)_{k t} \delta_{j i}+\left(\tilde{A}^{2}\right)_{j t} \delta_{i k}+\left(\tilde{A}^{2}\right)_{i t} \delta_{j k}$.

The proof now is a long computation using the transformation law for $\nabla_{g}^{2} \mathcal{A}_{g}$ and $\mathcal{A}_{g}^{2}$ under a conformal change of the metric (see e.g. [2]).

Proof of Corollaries 1.4 and 1.8 and of Proposition 1.10. On $\left(\mathbb{R}^{n}, g_{\text {flat }}\right)$ we have $Z_{g_{\text {flat }}} \equiv 0$, and the claim follows upon carefully rearranging terms on the righthand side of equation (1.7) in order to recover (a multiple of) $T_{4,(n-4) /(2 n)}^{u}$; see also Theorem 1.20 in [7] for the explicit expressions for $T_{4, \alpha}^{u}$ and $T_{2, \alpha}^{u}$. The same procedure can be applied to equation (1.10) in order to recover (1.11), also exploiting Theorem 1.13 in [6]. The proof of Proposition 1.10 follows from a similar, but longer, procedure.

Proof of Corollaries 1.5 and 1.9. We start from (1.7) and we set $i=j$ and $k=t$. Now we use the facts that $Z_{i i t t}=-Q_{g}$ and $u_{i i t t}=\Delta_{g}^{2} u$, and the well-known commutation relation

$$
u_{i j k}=u_{i k j}+u_{t} R_{t i j k}
$$

which implies

$$
u_{i t t}=u_{t i t}=u_{t t i}+u_{t} R_{t i} .
$$

Equation (1.9) can be now obtained through a careful computation exploiting the definitions of the Schouten tensor, the Paneitz operator and of the $Q$-curvature (see equations (1.1) and (1.2)). The same procedure can be applied to (1.10) in order to obtain equation (1.12).

Proof of Propositions 1.12 and 1.13. Since $(M, g)=\left(\mathbb{R}^{n}, g_{\text {flat }}\right)$, we have $\mathcal{A}_{g_{\text {flat }}} \equiv 0$. From the standard transformation law for the Schouten tensor and its covariant derivative under a conformal change of the metric (see e.g. [2]) we deduce (1.14), (1.15), (1.16) and (1.17). 
Acknowledgements. P. Mastrolia wishes to thank M. Salvatori, F. Gallo and J. E. Massetti for several stimulating discussions. D. D. Monticelli wishes to thank C. Bardelle, D. Lupo and K. R. Payne. The authors also wish to thank the referee for useful comments and remarks which improved the exposition of the results.

\section{References}

[1] Bensouf, A. And Chtioui, H.: Conformal metrics with prescribed $Q$-curvature on $S^{n}$. Calc. Var. Partial Differential Equations 41 (2011), no. 3-4, 455-481.

[2] Catino, G., Mastrolia, P., Monticelli, D. D. and Rigoli, M.: Conformal Ricci solitons and related integrability conditions. Preprint available at arXiv: 1405.3169v1 [math.DG], 2014.

[3] Djadli, Z., Hebey, E. And Ledoux, M.: Paneitz-type operators and applications. Duke Math. J. 104 (2000), no. 1, 129-169.

[4] Graham, C.R., Jenne, R., Mason, L. J. and Sparling, G. A. J.: Conformally invariant powers of the Laplacian. I. Existence. J. London Math. Soc. (2) 46 (1992), no. $3,557-565$.

[5] Li, A. AND Li, Y. Y.: On some conformally invariant fully nonlinear equations. Comm. Pure Appl. Math. 56 (2003), no. 10, 1416-1464.

[6] Li, Y. Y., Mastrolia, P. And Monticelli, D. D.: On conformally invariant equations on $\mathbb{R}^{n}$. II. Exponential invariance. Nonlinear Anal. 75 (2012), 5194-5211.

[7] Li, Y. Y., Mastrolia, P. And Monticelli, D. D.: On conformally invariant equations on $\mathbb{R}^{n}$. Nonlinear Anal. 95 (2014), 339-361.

[8] Li, Y. Y. And Monticelli, D. D.: On fully nonlinear CR invariant equations on the Heisenberg group. J. Differential Equations 252 (2012), no. 2, 1309-1349.

[9] Mastrolia, P., Rigoli, M. And Setti, A. G.: Yamabe-type equations on complete, noncompact manifolds. Progress in Mathematics 302, Birkhäuser/Springer Basel AG, Basel, 2012.

[10] Viaclovsky, J. A.: Conformal geometry, contact geometry, and the calculus of variations. Duke Math. J. 101 (2000), no. 2, 283-316.

Received February 7, 2013; revised November 27, 2013.

Paolo Mastrolia: Università degli Studi di Milano, Dipartimento di Matematica, via C. Saldini 50, 20133 Milano, Italy.

E-mail: paolo.mastrolia@unimi.it

Dario D. Monticelli: Università degli Studi di Milano, Dipartimento di Matematica, via C. Saldini 50, 20133 Milano, Italy.

E-mail: dario.monticelli@unimi.it

The two authors are supported by GNAMPA project "Analisi globale ed operatori degeneri" and are members of the Gruppo Nazionale per l'Analisi Matematica, la Probabilità e le loro Applicazioni (GNAMPA) of the Istituto Nazionale di Alta Matematica (INdAM). The first author was partially supported by FSE, Regione Lombardia. The second author was partially supported by GNAMPA project "Equazioni differenziali con invarianze in analisi globale" 